\title{
Calibration for Stochastic Existing Building Stock Model for Energy Simulation
}

\author{
Hye Gi Kim ${ }^{1}$, Sun Sook Kim² \\ ${ }^{1}$ Department of Architecture, Graduate School, Ajou University, Suwon, South Korea \\ ${ }^{2}$ Department of Architecture, Ajou University, Suwon, South Korea
}

\begin{abstract}
Building energy model (BEM) is useful in the retrofit stage of buildings because they allow flexible evaluation of various technology combinations or new technologies. However, the assumptions and uncertainties of the input variables produce a difference between the calculated energy and the measured energy. Old buildings or building stocks are more difficult to develop due to more assumptions and uncertainties. This paper describes a stochastic approach and a calibration method which can account different sources of uncertainty to develop an BEMs that can show energy consumption characteristics of existing building stock in Korea. First, described the process of deriving probability distribution and performing simulation using a database of nationwide building and energy information in Korea. Next, Inverse modelling was proposed to obtain parameters which is an auxiliary variable that describe the relationship between building performance and energy usage. The parameters both the outputs to the performance of the building or system and the input variables that determine energy usage at the same time. Therefore, using the parameters as information for calibration can improve the accuracy of the calibrated model, and identify the cause of inconsistencies with energy usage by ability to compare and calibrate the performance of a building or system in detail. Finally, calibration was performed, and the change of output was discussed.
\end{abstract}

\section{Introduction}

With the increasing needs for energy efficiency and management of existing buildings, the roles of energy performance analysis and assessment have also increased
(M.C. Kennedy, 2001). Simulation which is a typical building energy performance analysis tool, can consider the system variables, the physical characteristics of the building, and the functional nature, by modelling based on the heat transfer and thermodynamic relationships. In other words, simulation has the characteristic that it is possible to review new technology without relying on past data. However, it requires substantial time and effort in data collection, input and result output. In particular, there are large variations in data availability for existing buildings unlike new buildings. In addition, there are uncertain factors that can interfere with the building energy modelling, such as changes in thermal performance due to deterioration, randomness to the presence and behaviour of occupants, and stochastic changes in indoor and outdoor environmental conditions. Thus, a deterministic approach that a single arbitrary value is input following a case study, or a simulation is performed using reference building developed and provided at a national level is generally performed $(\mathrm{G}$. Augenbroe, 2002). As a result, a lot of uncertain inputs entered differently than actual are interacted with each other, resulting in a difference between actual energy consumption and simulation results. Such problem causes confusion in stakeholder investment decisions of energy improvement opportunities or the energy-efficient policies and programs.

For this reason, the calibration of BEMs is of growing interest. Model calibration is the process of improving the accuracy of BEMs to reflect actual operating conditions. Calibration can be classified by three general categories as shown in Table 1 (Yoon, 2014; Lim, 2017; Coakley, D., 2011 and 2014;).

Table 1. Types of model calibration methods

\begin{tabular}{|c|c|l|}
\hline \multicolumn{2}{|c|}{ Method } & \multicolumn{1}{c|}{ Characteristics } \\
\hline \multicolumn{2}{|c|}{$\begin{array}{c}\text { Trial-and-Error } \\
\text { (Manual) }\end{array}$} & $\begin{array}{l}\text { - Calibrate the input variable values of the building energy model through trial and error until the } \\
\text { output of the building energy model is similar to the actual building measurement data } \\
\text { - Highly dependent on the experience and expertise of the analysts performing the calibration } \\
\text { - Method can get calibration result desired but the solution that results may not be the true solution }\end{array}$ \\
\hline \multirow{2}{*}{$\begin{array}{c}\text { Computer } \\
\text { based } \\
\text { calibration }\end{array}$} & \begin{tabular}{l} 
Optimization \\
\cline { 2 - 4 }
\end{tabular} & $\begin{array}{l}\text { - Using a gradient-based algorithm to find the value of the input variable that minimizes the } \\
\text { difference between the output value of the building energy model and the measured data value } \\
\text { - The unknown variable is assumed to be a deterministic one, excluded the probabilistic nature }\end{array}$ \\
approach & $\begin{array}{l}\text { - Obtain a probability model of an input variable that reduces the error between the predicted value } \\
\text { and the actual value of the model } \\
\text { - By injecting a stochastic input variable, the result of the model is also computed as a probability } \\
\text { distribution }\end{array}$ \\
\hline
\end{tabular}

